\title{
Amano Lipase $A$ grafting onto a silica surface
}

\author{
JAKUB ZDARTA, TEOFIL JESIONOWSKI * \\ Faculty of Chemical Technology, Poznan University of Technology, Poznań, Poland \\ *Corresponding author: teofil.jesionowski@put.poznan.pl
}

\section{Introduction}

Since their discovery, enzymes have fulfilled an important role as catalysts of many chemical reactions. Their high selectivity, efficiency and their rate of catalyzing are the reasons for interest in the industrial utilization of biocatalysts. However, due to the low stability and resistance of enzymes in process conditions, this task proved to be very difficult to accomplish. Previous studies have resulted in the development of a number of methods, the aim of which has been the improvement of enzyme properties, and in particular immobilization that is based on the decreasing mobility of enzymes (Synowiecki and Wołowska, 2007). Substances obtained via this method can be characterized not only by an improved chemical and thermal stability, but they also show a slower decrease in catalytic activity in comparison to free enzymes. An additional advantage of immobilized biocatalysts is the possibility of performing catalyzed reactions in polar as well as non-polar solvents (Tischer and Wedekind, 1999).

Few immobilization methods are commonly applied, and they differ in terms of the supports utilized, the type and energy of interactions between the matrix and enzyme, and the type of compound participating in the formation of the interaction. Often, the type of reaction and the enzyme that catalyzes a given chemical change determine the choice of the immobilization method. Solutions already developed can be divided into immobilization with covalent bond formation, immobilization through encapsulation, entrapment and cross-linking (Kalantari et al., 2012). The most popular and commonly used method of enzyme immobilization is adsorption on a carrier. This procedure is based on the creation of specific interactions between the biocatalyst and the support, which usually are hydrogen bonds or van der Waals forces (Cao et al., 2003). Formation of these relations enables the application of an intermediate compound which most often is a representative of biofunctional carbonyl compounds, for example, glutaraldehyde. The advantages of this method are the lack of interference in the enzyme internal structure, the rate of the process and its low cost; however, the drawbacks include weak biocatalyst connections with the support, which may result in fast elution of the enzyme from the support, being a consequence of a loss of the catalytic activity of the substance.

This paper presents the results of studies undertaken to develop an effective adsorption immobilization procedure for Amano Lipase A on an inorganic support with the use of a glutar aldehyde as an intermediate compound in the creation of interactions between the silica support and the biocatalyst.

\section{Experimental}

\section{Silica preparation}

Silica was obtained via a modified Stöber method (Stöber et al., 1968). The process is based on hydrolysis and a tetraethoxysilane (TEOS) condensation reaction in ethanol, in the presence of ammonia as a reaction catalyst. The process was carried out in elevated temperatures for $30 \mathrm{~min}$. TEOS was dosed dropwise for $15 \mathrm{~min}$ into a mixture of ethanol and ammonia in 1:10 volume ratio. After precipitation, silica was filtered under reduced pressure and dried at $105^{\circ} \mathrm{C}$. The obtained $\mathrm{SiO}_{2}$ powder underwent an Fourier transform infrared spectroscopy (FT-IR) analysis, a particle size distribution analysis and morphological characterization.

\section{Grafting of glutaraldehyde onto silica surface}

After weighing an apropriate amount of silica, 6\% glutaraldehyde solution in phosphate buffer of a $\mathrm{pH}$ equal to 7 was added. Once prepared, the mixture was shaken in a shaker at room temperature. When the process was finished the mixture was filtered under reduced pressure, dried at a temperature of about $105^{\circ} \mathrm{C}$ for $24 \mathrm{~h}$ and subjected to an FT-IR analysis. 


\section{Adsorption immobilization of Amano Lipase $A$}

Immobilization was performed analogous to the procedure proposed by Gustafsson et al. (Gustafsson et al., 2012). According to this method, phosphate buffer and Amano Lipase $A$ solution of an appropriate concentration in the same buffer was added to the modified silica with glutaraldehyde,. The mixture was shaken for $96 \mathrm{~h}$ in a shaker at room temperature. When the process was finished the mixture was filtered under reduced pressure, and the support with the introduced enzyme was dried at room temperature for about $24 \mathrm{~h}$. The samples were evaluated applying FT-IR and thermogravimetric analyses. The parameters of the porous structure were also examined.

\section{Results and discussion}

A dispersive-morphological analysis of silica dioxide (IV) was carried out based on photographs performed with the use of an EVO 40 scanning electron microscope (SEM) made by Zeiss, and the particle size distribution was estimated with a Zetasizer Nano ZS by Malvern Instruments Ltd. The model microphotograph, presented in Figure 1, shows that the obtained silica can be characterized with spherical particles of about $500 \mathrm{~nm}$ in diameter. Results of the particle size analysis aren presented in the form of a volumetric fraction size distribution (Fig. 2). Analyzing the obtained data it was found that particles were uniform, with a size range from $342 \mathrm{~nm}$ to $825 \mathrm{~nm}$, and the biggest volume fraction $(33.2 \%)$ was characteristic of particles of $531 \mathrm{~nm}$ in diameter. The obtained data were confirmed by observation performed with the use of an SEM technique.

FT-IR analysis was used to confirm the silica surface modification with glutaraldehyde. The obtained FT-IR

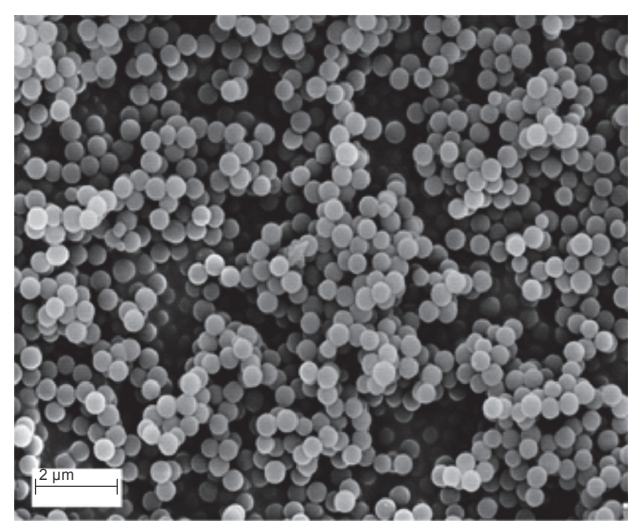

Fig. 1. SEM image of the obtained silica

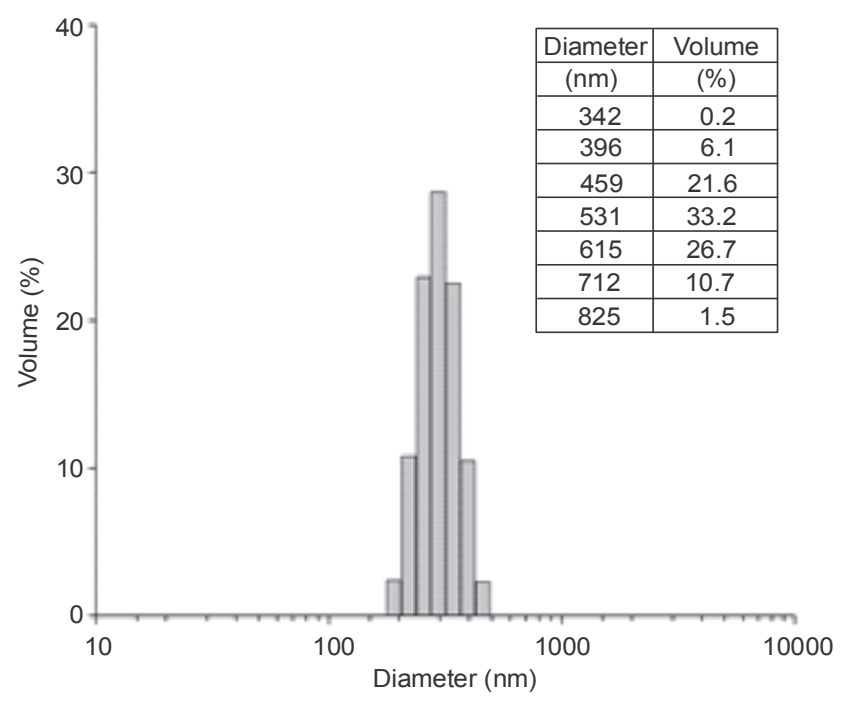

Fig. 2. Particle size distribution by volume contribution for $\mathrm{SiO}_{2}$ support

spectrum was compared with a spectrum of unmodified silica and is presented in Figure 3. In the spectrum of non-functionalized silica characteristic signals can be observed: they originated from silanol groups $\equiv \mathrm{Si}-\mathrm{OH}$ occuring at a wavenumber equal to $3748 \mathrm{~cm}^{-1}$, and a signal at $1120 \mathrm{~cm}^{-1}$ which comes from stretching vibrations of $\equiv \mathrm{Si}-\mathrm{O}-$. The remaining 3 signals appearing between 1000 and $469 \mathrm{~cm}^{-1}$ are related to asymmetric and symmetric bending and stretching vibrations which are generated by the $\equiv \mathrm{Si}-\mathrm{O}-\mathrm{Si} \equiv$ group. The band between $3500 \mathrm{~cm}^{-1}$ and $3200 \mathrm{~cm}^{-1}$, which refers to stretching vibrations of $-\mathrm{OH}$, is also visible. Compared to the spectrum of unmodified silica, which is in the spectrum of support after functionalization, the intensity of the signal characteristic of glutaraldehyde increased, covering bands related to the support. This means that the silica surface was succesfully modified with glutaraldehyde.

Figure 4 shows FT-IR spectra of Amano Lipase $A$ (ALA) set-up with spectra of the support after the enzyme immobilization process carried out for $24 \mathrm{~h}$ (ALA 24) and $96 \mathrm{~h}$ (ALA 96). In the spectrum of native enzymes, a series of signals characteristic of surface groups of the protein is clearly visible. Among these signals, one at a wavenumber around $2900 \mathrm{~cm}^{-1}$ originating from stretching vibrations of the $\equiv \mathrm{C}-\mathrm{H}$ group is particularly distinguishable. Additionally, wide and intensive bands are easily noticable between $3600 \mathrm{~cm}^{-1}$ and $3200 \mathrm{~cm}^{-1}$ originating from stretching vibrations of the $-\mathrm{OH}$ group, which mask the stretching vibrations band of the $-\mathrm{NH}$ group. Characteristic signals originating from $\equiv \mathrm{C}-\mathrm{O}-$ 


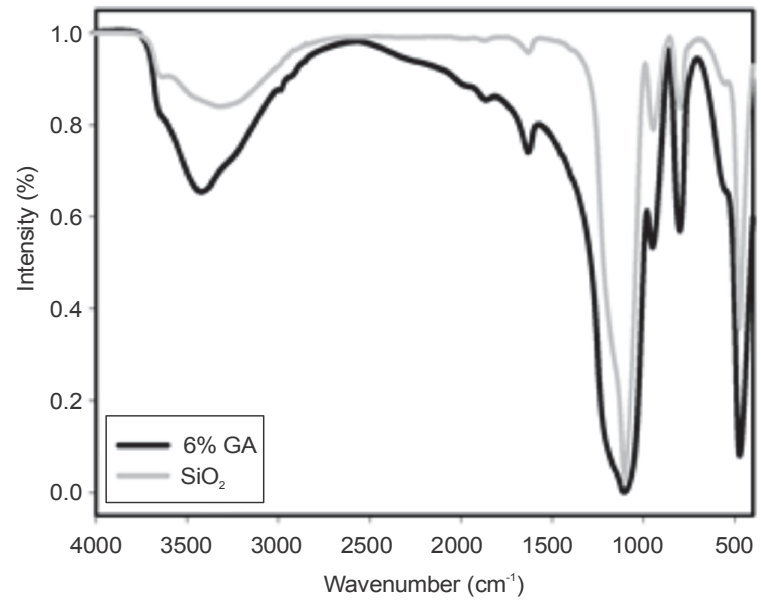

Fig. 3. FTIR spectra of unmodified silica grafted with glutaraldehyde

group stretching vibrations are noticeable at wavenumbers equal to $1700 \mathrm{~cm}^{-1}$ and $1180 \mathrm{~cm}^{-1}$. Deformed vibrations originating from $-\mathrm{NH}$ and $-\mathrm{OH}$ groups are visible near $1550 \mathrm{~cm}^{-1}$ and $1400 \mathrm{~cm}^{-1}$, respectively. Moreover, in the range below $1000 \mathrm{~cm}^{-1}$ a series of signals related to various vibrations of the $\equiv \mathrm{C}-\mathrm{C} \equiv$ group appear.

The set-up of spectroscopic analyses presented below confirms effective immobilization of a biocatalyst on the surface of the support. In the spectrum of a sample after immobilization, not only signals characteristic of silica are visible, but also the spectra originating from groups located at the surface of the enzyme.

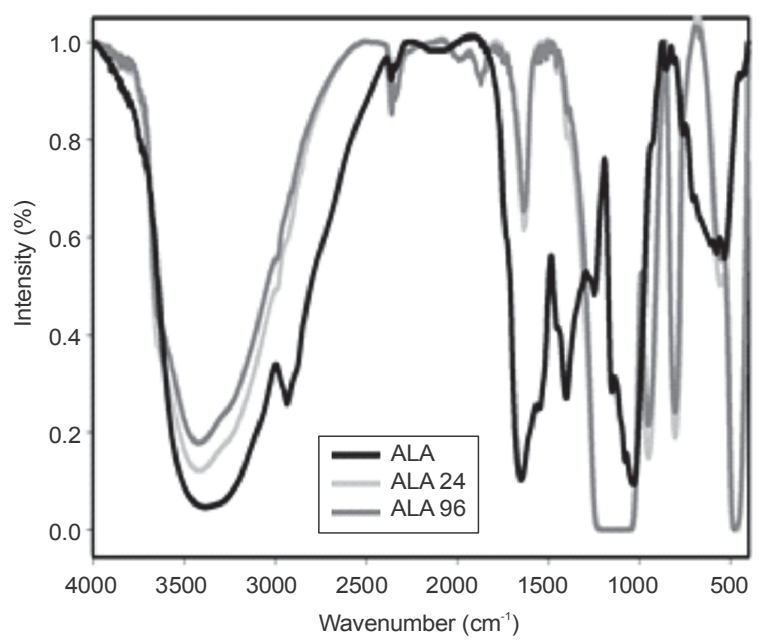

Fig. 4. FTIR spectra of the silica after immobilization of Amano Lipase A for $24 \mathrm{~h}$ and $96 \mathrm{~h}$

Additional confirmation of a succesfully conducted immobilization process are the results obtained from the adsorption analysis presented in Table 1 . In comparison to the data characteristic of the support, after immobilization all the parameters describing the obtained product decreased, which confirms accomplishment of the enzyme immobilization process.

Table 1. Parameters of the porous structure of an unmodified $\mathrm{SiO}_{2}$ and silica after immobilization

\begin{tabular}{l|c|c}
\hline \multicolumn{1}{c|}{ Analyzed parameter } & Silica & $\begin{array}{c}\text { Silica after } \\
\text { immobilization }\end{array}$ \\
\hline BET surface area $\left[\mathrm{m}^{2} / \mathrm{g}\right]$ & 6.12 & 4.98 \\
\hline Pore size $[\mathrm{nm}]$ & 3.25 & 2.96 \\
\hline Pore volume $\left[\mathrm{cm}^{3} / \mathrm{g}\right]$ & 0.005 & 0.003 \\
\hline
\end{tabular}

\section{Conclusions}

The obtained silica can be characterized by uniform, spherical particles of a size of about $500 \mathrm{~nm}$, which enables fast and effective surface modification with the use of a glutar aldehyde. FT-IR spectroscopy and porous strtucture analysis confirmed an appropriate introduction of a bifunctional carbonyl compound which participates in the formation of interactions between the enzyme and the support, as well as an Amano Lipase A adsorption immobilization process.

The development of a simple and effective procedure for an immobilization process opens many possibilities for its use in important catalytic applications. The optimalization of the presented method enables not only an increase in the introduced amount of enzyme, but also better utilization of the substances used in the process.

\section{Acknowledgements}

This work was supported by Poznan University of Technology, research grant no. 32-375/2013-DS.

\section{References}

Synowiecki J., Wołowska S. (2007) Biotechnology 2(77): 7-26. Tischer W., Wedekind F. (1999) Top. Cur. Chem. 200: 96-126. Kalantari M., Kazemeni, M., Tabandeh F., Arpanaei A. (2012) J. Mater. Chem., 22: 8385-8393.

Cao L., Van Langen L., Sheldon R.A. (2003) Curr. Opin. Biotechnol. 14: 387-394.

Stöber W., Fink A., Bohn E. (1968) J. Colloid Interface Sci. 26: 62-69.

Gustafsson H., Johansson E.M., Barrabino A., Oden M., Holmberg K. (2012) Colloids Surf. B 100: 22-30. 